\title{
"Zeltdörfer im Burgenland" \\ Zur Relevanz materieller und sprachlicher Bilder im politischen Diskurs. Eine exemplarische Analyse
}

\author{
Dorothee Meer (Bochum)
}

\begin{abstract}
This paper analyses the use of figures of speech as well as of material images from a discourse semiotic point of view. The empirical basis comprises the news coverage of the $F A Z$ and of the $S Z$ concerning the hunger strike of refugees on the Munich Rindermarkt during the summer of 2013. Based on this specific empirical data, this paper initially illustrates basic assumptions with regard to the specific semiotic resources of figures of speech and of material images. Subsequently, it delineates different strategies of using both kinds of images by contrastively analysing both newspapers. The paper's conclusion, then, presents an evaluation of particular recommendations for the purposeful use of images in political contexts.
\end{abstract}

\section{$1 \quad$ Einleitung}

Mit einem Großaufgebot hat die Polizei am Sonntag ein Zeltlager am Münchener Rindermarkt geräumt, in dem Asylbewerber mit einem Ess- und Trinkstreik ein unbefristetes Aufenthaltsrecht erzwingen wollten. 350 Beamte umstellten am Morgen gegen fünf Uhr das Lager, das die Flüchtlinge und Unterstützer errichtet hatten. 44 Personen, unter ihnen drei Kinder, die nach Einschätzung der Behörden teilweise in Lebensgefahr schwebten, wurden zur ärztlichen Versorgung in Krankenhäuser gebracht. Unterstützer versuchten mit Sitzblockaden das Vorgehen der Polizei und der Rettungskräfte zu stören. Dreizehn Personen wurden festgenommen, unter ihnen ein Mann, der als Vertreter der Asylbewerber aufgetreten war, aber nicht an dem Ess- und Trinkstreik teilgenommen hatte.

(Schäffer 2013a: 2)

Mit diesen Worten beschrieb die Frankfurter Allgemeine Zeitung (FAZ) am 01.07.2013 das Ende eines achttägigen Hungerstreiks von Flüchtlingen am Münchener Rindermarkt. Den Flüchtlingen ging es mit ihrer Aktion vom 22. bis zum 30.06.2013 neben dem Erlangen ihres Bleiberechts um eine Verbesserung der politischen Situation von Flüchtlingen in Deutschland, speziell in bayerischen Aufnahmelagern.

Schaut man sich die Meldung der FAZ genauer an, so ergeben sich ausgehend von der sprachlich realisierten Situation eine Reihe anschaulicher Bilder. Diese vermitteln Leser/inne/n eine unmittelbare bildliche Vorstellung des beschriebenen Polizeieinsatzes. Diese Vorstellung kann im Anschluss an Ulla Fix (2001: 11-12) als mentaler Bildkomplex beschrieben werden. Sie setzt sich im vorliegenden Fall zusammen aus Bildern des Abtransports hungernder, ver- 
mutlich widerstandslos apathischer Flüchtlinge einerseits und der Verhaftung widerständiger Unterstützer andererseits, die (möglicherweise mit Gewalt) von den Einsatzkräften der Polizei verhaftet werden. Eine Sonderstellung bildlicher Vagheit nimmt im skizzierten Szenario die Verhaftung des "ein[en] Mann[es]" ein, der als "Vertreter der Asylbewerber" vorgestellt wird. Dieser Mann stellt auf der Ebene der Anschaulichkeit zunächst eine bildliche Leerstelle dar.

Ausgehend von dieser ersten Skizze lässt sich die doppelte Fragestellung des vorliegenden Beitrags verdeutlichen: Zum einen soll am Beispiel des angesprochenen Hungerstreiks auf dem Münchener Rindermarkt danach gefragt werden, wie sprachliche und materielle Bilder des konkreten Ereignisses aus diskurssemiotischer Sicht im Rahmen der medialen Berichterstattung interagieren, zum anderen soll aus diskursanalytischer Perspektive untersucht werden, wie sich die Wahrnehmung der Hungerstreikenden im Rahmen der Berichterstattung der Süddeutschen Zeitung (SZ) und der Frankfurter Allgemeinen Zeitung unterscheidet. Mit dieser doppelten Perspektive geht es entgegen der lang anhaltenden linguistischen "Blindheit für Bilder" (vgl. Schmitz 2005) darum, einerseits sprachliche Bilder in ihrer Funktion im politischen Diskurs exemplarisch zu betrachten, andererseits materielle Bilder (hier: journalistische Fotografien) sowohl in ihrer semiotischen Autonomie als auch als Teil des konkreten "Sprache-Bild-Diskurses" zu begreifen. Vor dem Hintergrund des Rahmenthemas dieses Heftes "Sprache und Demokratie" besteht ein hieran anschließendes weiterführendes Ziel des Beitrags darin, die diskurstheoretischen Befunde gleichzeitig diskurspraktisch zu nutzen, indem ihr Handlungswert für den Bereich politischer Gegenbewegungen zur aktuellen Flüchtlingspolitik herausgestellt wird. Vor allem ausgehend vom letztgenannten Aspekt wird deutlich, dass es insgesamt darum geht, die sprachlichen und bildlichen Partizipationsmöglichkeiten von Randgruppen analytisch in den Blick zu nehmen, um hierauf aufbauend gegenhegemoniale diskursive Strategien ableiten zu können.

Aus empirischer Sicht stütze ich mich auf die Berichterstattung der Süddeutschen Zeitung und der Frankfurter Allgemeinen im Zeitraum des Hungerstreiks. Konkret handelt es sich hierbei um die folgenden Ausgaben der beiden Zeitungen:

- $\quad$ Süddeutsche Zeitung (SZ) online vom 22.06.2013-29.06.2013

- Süddeutsche Zeitung (offline) vom 24.06.-02.07.2013

- Frankfurter Allgemeine (FAZ) online vom 29.-31.06.2013

- Frankfurter Allgemeine (offline) vom 01.07.2013-31.07.2013. ${ }^{1}$

Es handelt sich somit um eine (kleine) exemplarische Studie, deren Vorteil in der relativen Abgeschlossenheit des Ereignisses liegt. So stellt gerade die Begrenztheit des Untersuchungsgegenstands für eine erste Hypothesenbildung hinsichtlich des Gebrauchs von Bildern unterschiedlicher semiotischer Qualität einen Vorteil dar.

\footnotetext{
1 Die mediale Differenzierung zwischen der online- bzw. der offline-Version der jeweiligen Zeitungen ist für die folgenden Überlegungen irrelevant. Zitate aus der offline-Version einer der beiden Zeitungen werden nicht explizit markiert. Zitate aus den online-Ausgaben werden entweder über die Angabe der Uhrzeit (SZ) oder über die Angabe "(online) (FAZ)" kenntlich gemacht.
} 
Im folgenden Abschnitt (Kapitel 2) sollen unter Nutzung des konkreten empirischen Materials einige grundlegende Annahmen bezüglich der unterschiedlichen semiotischen Ressourcen von materiellen und sprachlichen Bildern aufgezeigt werden. Hieran anschließend (Kapitel 3) geht es darum, das diskursive Ereignis ${ }^{2}$ des Hungerstreiks durch die kontrastive Analyse der Berichterstattung in der $F A Z$ und der $S Z$ hinsichtlich der genutzten semiotischen Strategien näher zu beschreiben. Im letzten Schritt (Kapitel 4) sollen diese Befunde diskurssemiotisch ausgewertet und im Hinblick auf konkrete politische Anschlussüberlegungen genutzt werden.

\section{Sprachliche und materielle Bilder am Beispiel des Hungerstreiks}

\subsection{Sprachliche Bilder im politischen Diskurs}

Zunächst einmal geht es unter Bezug auf das konkrete diskursive Ereignis des Hungerstreiks darum, aus semiotischer Sicht zwischen materiellen und sprachlichen Bildern zu differenzieren (vgl. Fix 2001: 10; Stöckl 2004: 11-42). Zu diesem Zweck soll auf einen Auszug aus einem Kommentar der $S Z$ zurückgegriffen werden, der am 02.07.2013, zwei Tage nach der Auflösung des Hungerstreiks unter der Überschrift "Im Burgen-Land" zu lesen war:

In der Münchener Innenstadt war eine Woche lang eine Attraktion der besonderen Art zu besichtigen. Eine Burg stand da errichtet aus Pavillons und Plastikplanen, darin Flüchtlinge im Durststreik. Viele Münchener haben geschimpft über den Verhau und die frechen Forderungen der Flüchtlinge, die bleiben wollen. Dabei haben die Bürger vergessen, dass sie selbst in einer Burg leben, einer viel komfortableren, bewehrt mit Mauern aus Paragraphen. Diese Burg nennt sich Europa.

(Kastner 2013a: 3)

Wie bei dem bereits zitierten Zeitungsbericht aus der $F A Z$ handelt es sich auch bei diesem Kommentar der $S Z$ um eine anschauliche Situation. Diese ergibt sich an einigen Stellen aus der Bedeutung der gewählten Wörter: "besichtigen" "Münchener Innenstadt", "Attraktion besonderer Art", "Flüchtlinge im Durststreik", "Verhau". Die genannten Lexeme und Lexemkombinationen ermöglichen es, dass wir "uns im Wortsinn etwas" vorstellen (Fix 2001: 18). Letzteres gilt zwar auch für die Syntagmen "eine Burg stand da errichtet aus Pavillons und Plastikplanen, darin Flüchtlinge im Durststreik", die Bürger leben "selbst in einer Burg" und "[d]iese Burg nennt sich Europa", aber in diesen Syntagmen wird der Aspekt der unmittelbaren Anschaulichkeit, der sich aus Lexemen wie "Burg" oder "Pavillons und Plastikplanen" ergibt, ergänzt durch metaphorische Prozesse der Übertragung (vgl. Fix 2001: 13, 19). So wird zum einen das Konzept der mittelalterlichen Burg auf das Lager der Flüchtlinge übertragen, indem die semantischen Merkmale Wehrhaftigkeit, Mächtigkeit und Abschottung des Lexems Burg zu den semantischen Merkmalen der instabilen Zelte der Hungerstreikenden

\footnotetext{
2 Unter einem diskursiven Ereignis möchte ich im Anschluss an Michel Foucault im Weiteren ein Ereignis von besonderem Gewicht verstehen, das durch das Zusammenkommen zufälliger und nicht-zufälliger Entwicklungen diskursiv wahrnehmbar und "auf der Ebene der Materialität wirksam" wird, ohne selbst materieller Natur zu sein (Foucault 1977: 39-40). Dass der Hungerstreik am Münchener Rindermarkt tatsächlich die Qualität eines diskursiven Ereignisses erlangte, hatte seinen Grund vor allem im Zusammenkommen des gewählten Ortes (dem "Herz" der bayerischen Hauptstadt), der kontinuierlichen überregionalen Berichterstattung der Süddeutschen Zeitung, mehreren zeitgleichen Krisen in der bayerischen Innenpolitik, den anstehenden Landtagswahlen in Bayern und der konkreten Aktion der Hungerstreikenden.
} 
(instabil, schutzlos, wehrhaft) hinzugefügt werden. Durch die Kombination beider Semkomplexe wird die Mächtigkeit und Anstößigkeit des Hungerstreiks in "der Münchener Innenstadt" unterstrichen. Zum andern wird das Bild der "Burg" auf die Situation der etablierten Münchener Bürger übertragen, deren "Burg" als "mächtiger" und "wehrhafter" charakterisiert wird, da sie zwar weniger sichtbar ist ("Mauern aus Paragraphen"), gleichzeitig jedoch umso umfassender ("Burg Europa"). Bei letzterem handelt es sich zusätzlich um ein verfremdendes Spiel mit der etablierten Metapher der "Festung Europa".

Die damit im Vorhergehenden von Fix (2001: 18-19) übernommene Unterscheidung zwischen (bildlicher) "Anschaulichkeit durch Wortbedeutung" und (bildlicher) "Anschaulichkeit durch [metaphorische] Verallgemeinerung" soll nun im nächsten Schritt durch den von Jürgen Link eingeführten Begriff des (Kollektiv-)Symbols ergänzt werden, der es erlaubt, die bisher beschriebenen Phänomene sprachlicher Bildlichkeit zu integrieren und für politische Kontexte nutzbar zu machen. Link (1997: 25) subsumiert unter der Begrifflichkeit der Kollektivsymbolik "die Gesamtheit der so genannten 'Bildlichkeit' einer Kultur, die Gesamtheit ihrer am weitesten verbreiteten Allegorien und Embleme, Metaphern, Exempelfälle, anschaulichen Modelle und orientierenden Topiken, Vergleiche und Analogien" und fasst das einzelne Kollektivsymbol als komplexe Vereinigungen von einem oder mehreren bildlichen Elementen, der Pictura (P), mit einem oder mehreren Sinnelementen, der Subscriptio (S) (vgl. Link 1984: 7, 1985: 168-169; Link/Paar 1990: 115). Überträgt man diese Definition auf den angeführten Kommentar der $S Z$, so ergibt sich folgende tabellarische Darstellung der Symbolstruktur.

\begin{tabular}{|c|c|}
\hline Pictura & Subscriptio \\
\hline & \\
\hline Burg (1), aus Pavillons und Plastikplanen & 'klein', 'wehrlos', 'instabil', 'ärmlich' \\
\hline Flüchtlinge im Durststreik & 'existenzielle Notsituation', 'politische Forderungen' \\
\hline Verhau & 'instabil', 'ärmlich', 'provisorisch' \\
\hline Burg (2) & 'stabil', 'geschützt', 'komfortabel', \\
\hline Mauer aus Paragraphen & 'gesetzlich geschützt' \\
\hline Burg (2) (Festung) Europa & 'territorial ausgedehnt', 'reich', 'legitim' \\
\hline
\end{tabular}

Tabelle 1: Quelle: Eigene Darstellung

Ausgehend vom vorliegenden Beispiel eines Zeitungskommentars werden einige Vorteile des Symbolkonzeptes deutlich: Zunächst einmal ist der Begriff des Symbols kompatibel mit einer Vielzahl traditioneller Tropen, von denen die Metapher, wie Fix (2001: 18) zu Recht betont, nur ein Mittel ist, Anschaulichkeit sprachlich darzustellen. Hieran anschließend erlaubt es der Begriff des Symbols zweitens - wie die Analyse der Metapher der "Burg" oben zeigt - die relevanten semantischen Operationen metaphorischer Übertragungsprozesse zu integrieren. Insoweit arbeitet der Symbolbegriff in doppelter Hinsicht textsensitiv, indem er sowohl die Picturae als auch die Subscriptiones analytisch aus dem Text gewinnt. Drittens, und das ist im Weiteren entscheidend, ermöglicht das Konzept nicht nur die Erfassung des Sinns von Elementen der Pictura durch die Bestimmung der entsprechenden Subscriptiones, sondern auch die Kopplung von Subscriptiones an bildliche Elemente, die nicht explizit realisiert werden. Hierfür ist das Beispiel der "Attraktion der besonderen Art" im zitierten SZ-Beitrag ein Beispiel: Obgleich hier nur die Subscriptio textuell realisiert wird, evoziert sie auf der Ebene der (stereotyp verankerten) mentalen Bilder Picturae wie "Schauspiel", "Zirkus" oder "Posse". 
Damit kommt als vierter Vorteil der Aspekt der kollektiven Verankerung von (Kollektiv)Symbolen in den Blick. So geht Link (1984: 69-70) davon aus, dass Symbole als Netze bzw. Gitter von Picturae und Subscriptiones im kollektiven Bewusstsein einer Kultur repräsentiert werden und bereits die Nennung des einen bzw. anderen Elements innerhalb des Netzwerkes verbundene weitere Elemente aufruft. Dies gilt, wie gerade gezeigt, aber nicht nur für Elemente der Subscriptio, sondern auch für bildliche Elemente, die als "Anschaulichkeit durch Wortbedeutung" klassifiziert werden können (vgl. Fix 2001: 18). So kann die Nennung der "Münchener Innenstadt" im oben zitierten Kommentar der $S Z$ sofort mit den Subscritiones 'finanzstark', 'sauber', 'ordentlich', 'gesetzeskonform' und 'weltoffen' verbunden werden.

Diese Annahmen werden durch den Text des zitierten Kommentars auch dadurch bestätigt, dass es die Binäropposition zwischen 'gesetzeskonform' (Pictura: "viele Münchener [...] haben geschimpft") und 'rechtswidrig' (Pictura: "Verhau", Subscriptio: "freche Forderungen") war, die dem Hungerstreik auf dem Münchener Rindermarkt seinen Charakter eines diskursiven Ereignisses gab. Im Vorgriff auf die Relevanz der (stereotypen) Verankerung materieller Bilder stellt hierbei die bildliche Vorstellung eines "Verhau[s]" ("aus Pavillons und Plastikplanen") in der Wahrnehmung "viele[r] Münchener" einen optimalen Kontrast dar, wie die beiden folgenden Bilder exemplarisch unterstreichen.

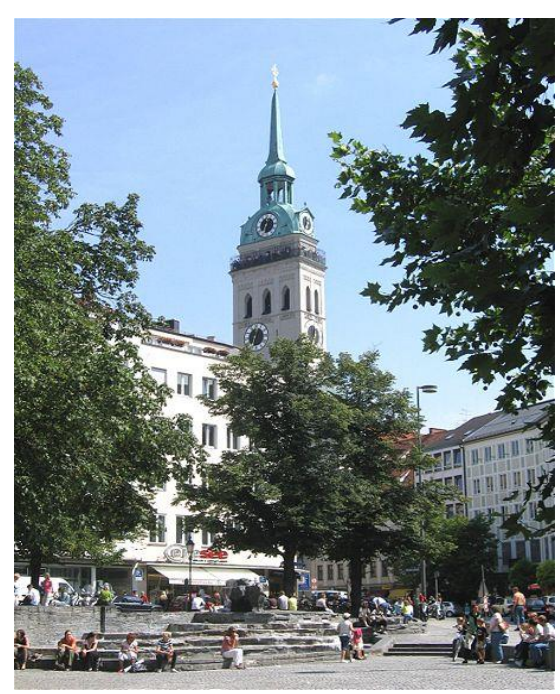

Abbildung 1: "Münchener Rindermarkt"

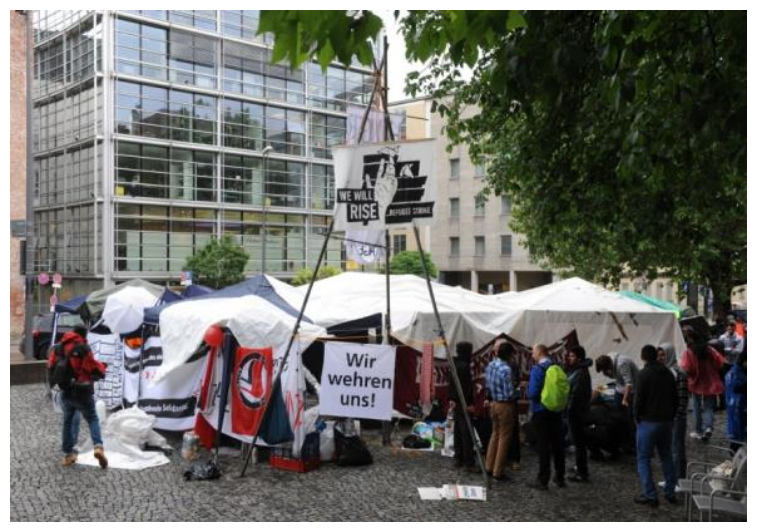

Abbildung 2: "Im Regen stehen gelassen"

Im Anschluss an Link soll bereits an dieser Stelle herausgestellt werden, dass verbale und materielle Bilder im kollektiven Unbewussten einer Kultur durch die Verknüpfung kollektiver 
Pictura- und Subscriptio-Netze abgesichert werden, eine Annahme, die Stöckl (2011: 48) aus einer anderen Perspektive durch den Hinweis bestätigt, dass "Sprachgebrauch und Bildgebrauch [...] in der mentalen Repräsentation zusammen[fallen], indem sie auf gleiche Invarianten ('Propositionen', 'Scripts', 'Frames', 'mentale Modelle' usw.) zurückgreifen". Ausgehend von dieser Feststellung soll im nächsten Abschnitt der Frage nach der Spezifik materieller Bilder im Rahmen des politischen Diskurses nachgegangen werden.

\subsection{Materielle Bilder im politischen Diskurs}

Die Spezifik materieller Bilder soll am Beispiel des in Abbildung 3 gezeigten Fotos der afghanischen Familie Wahafi (SZ) genauer betrachtet werden.

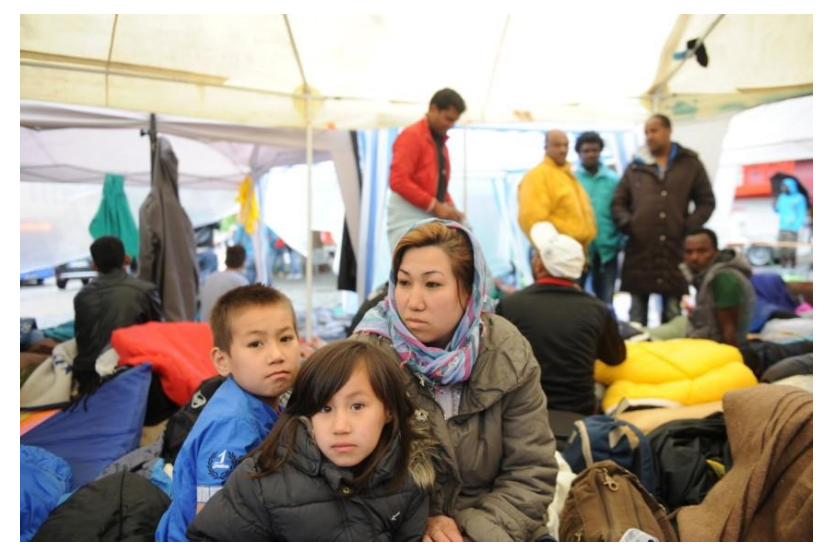

Abbildung 3: $S Z$ 25.05.2013, 08:53 Uhr

Zu Recht stellt Hartmut Stöckl (1998: 76, 2011: 48-49) immer wieder heraus, dass sich die Spezifik materiell-bildhafter Zeichenkomplexe deutlich von der Spezifik sprachlicher Zeichen unterscheidet und insoweit beide Zeichentypen zunächst in ihren unterschiedlichen semiotischen Eigenheiten wahrgenommen werden müssen, bevor ihr Zusammenspiel in komplexen Sprache-Bild-Texten erfasst werden kann. Hierauf aufbauend geht er davon aus, dass Bilder im Gegensatz zu sprachlichen Zeichen intentional offen sind, da sie zwar einen deskriptiven Inhalt haben, aber nichts behaupten können bzw. keine klar bestimmbare Aussage haben (vgl. Stöckl 1998: 77, 2011: 50), dass sie durch ihre fehlende deiktischen Verankerung vage, und in der Folge polyfunktional sind (vgl. Stöckl 1998: 77, 2011: 50) und dass sie aufgrund der Spezifik ihrer neurophysiologischen Verarbeitung schneller erfasst, länger behalten und leichter erinnert werden (vgl. Stöckl 1998: 77, 2011: 49). Darüber hinaus bewirke die (ikonische) Wahrnehmungsnähe von Bildern und ihre direkte neurologische Verarbeitung anders als bei sprachlichen Zeichen eine unmittelbar emotionale Wirkung (vgl. Stöckl 2011: 49-50). ${ }^{3}$

Bezieht man diese Überlegungen auf die vorhergehende Fotografie, ohne den Begleittext zu beachten, so bestätigen sich diese Annahmen hinsichtlich der intentionalen Offenheit, der Vagheit der Aussage und der fehlenden deiktischen Verankerung materiell-bildlicher Zeichen: Rein deskriptiv lässt sich zunächst einmal nur festhalten, dass es sich um die Aufnahme einer sitzenden Frau und zweier Kinder vor dem Hintergrund einer Vielzahl anderer Menschen handelt. Weiter kann aufgrund der Schlafsäcke und des Zeltdachs vermutet werden,

3 Als umfassende, tabellarische Zusammenfassung der spezifischen Potenziale bildlicher Zeichen siehe Stöckl (2011: 52). 
dass es sich um eine Art Lager handelt. Der statische Gesichtsausdruck der drei Personen im Vordergrund legt zusätzlich die Annahme nahe, dass die drei Personen sich in keiner zufriedenstellenden Situation befinden. Weitere kontextuelle Verankerungen enthält das Foto nicht. Doch auch ohne diese Kontexte zu kennen, prägt sich die Szene ein und löst vor allem aufgrund des emotionslosen Gesichtsausdrucks der Frau und der Kinder Empathie aus.

An dieser Stelle setzen Kress und van Leeuwen (1996: 1-2) an und weisen darauf hin, dass materiell-bildliche Darstellungen einer formalen "grammar of visual design" folgen. Aus dieser Bildgrammatik soll im vorliegenden Zusammenhang nur die Unterscheidung von drei Funktionsebenen genutzt werden, die die Autoren (vgl. Kress/van Leeuwen 1996: 41-42) im Anschluss an Halliday als "metafunctions" bezeichnen. Diese Metafunktionen sehen Kress und van Leeuwen (1996: 42-43) in der "ideational metafunction", die im Anschluss an Stöckl (2011: 51) mit "Darstellungsfunktion" oder "Ebene der Darstellung" übersetzt werden soll. Unter dieser Funktion verstehen die Autoren die Fähigkeit von Bildzeichen, Objekte der realen Welt bildlich zu realisieren und sie dabei in einer spezifischen und nicht zufälligen Art zueinander in Beziehung zu setzen. Als zweite Funktionsebene nennen die Autoren die "interpersonal metafunction", die im Anschluss an Jewitt und Oyama (2001: 145-146) als Interaktionsfunktion oder Ebene der Interaktion bezeichnet werden kann. Hierunter ist die Fähigkeit materiell-bildlicher Zeichen $\mathrm{zu}$ verstehen, einen Kontakt zwischen Zeichenproduzent/inn/en und ihren Rezipient/inn/en aufzubauen. Als dritte Funktion nennen Kress und van Leeuwen (1996: 43-44) die "textual metafunction", die mit "Textualitätsfunktion" oder "Ebene der Vertextung" wiedergegeben werden soll. Hierunter ist die Fähigkeit visueller Zeichenelemente zu begreifen, kohärente Texte herzustellen.

Hierauf aufbauend lässt sich im Hinblick auf das Foto in Abbildung 3 auf der Ebene der Darstellung festhalten, dass die Abbildung von zwei Kindern, die vor einer Frau hocken, die stabile Annahme evoziert, dass es sich um eine Mutter mit ihren Kindern handelt. Ebenso lassen der emotionslose Gesichtsausdruck aller drei Protagonisten und die Dominanz von beigen, braunen und grauen Tönen vermuten, dass sich die dargestellten Personen in einer prekären Situation befinden. Weiter legt das Setting des "Lagers" die Annahme nahe, dass es sich um Flüchtlinge handelt, da alle erkennbaren Personen (aus ethnischer Perspektive) nichtdeutscher Herkunft sind. Die zufällige Vermischung von Menschen unterschiedlicher Nationalitäten auf der Flucht wird auch durch einige grelle Farbtupfer vor tristem Hintergrund als multi-ethnisch unterstrichen. Lagerbilder wie diese werden medial immer wieder verbreitet, sodass sie jenseits des konkreten Kontextes "wieder"erkannt und inhaltlich gelesen werden können.

Jenseits der Darstellung des Settings verdeutlicht die Positionierung der Flüchtlinge unterhalb des geometrischen Mittelpunkts in Kombination mit der frontalen Aufnahme des Fotos, dass Rezipient/inn/en die Dreiergruppe zwar direkt wahrnehmen, in Kontakt mit ihnen treten (Interaktionsfunktion), die drei Personen jedoch gleichzeitig als Teil der Gesamtszenerie lesen, als Exempel (Vertextungsfunktion). Diese Wirkung auf der Ebene der Vertextung wird zusätzlich dadurch hervorgerufen, dass der Hintergrund der Aufnahme eine stärkere Unschärfe aufweist als die drei "Hauptpersonen" im Vordergrund.

Trotz dieser Erkenntnisse ist die präzise Bestimmung des Settings, die Herstellung lokaler, temporaler und kausaler Eindeutigkeit, aber auch die konkrete (politische) Positionierung der 
Szene, erst durch ergänzende Formen der sprachlichen Kommentierung möglich. Diese Überlegung leitet über zur Feststellung Stöckls (2011: 48), dass materielle und verbale Bilder sich gerade aufgrund ihrer unterschiedlichen Ressourcen gegenseitig ergänzen und im Rahmen von Sprache-Bild-Texten komplexe Einheiten eingehen.

\subsection{Sprache-Bild-Texte im politischen Diskurs}

Unabhängig von semiotischen Überlegungen ist es aus praktischer Sicht unmittelbar evident, dass materielle Bilder nahezu nie ohne sprachliche Kontexte auftreten. Selbst in Museen, dem prototypischen Ort der Ausstellung von Bildern, ergänzen die Namen der Maler/innen, die Titel der Bilder und weitere Erklärungen den Kontext des präsentierten Exponats sprachlich (heute z. B. häufig in Form von "Audio-Guides"). Zu dieser Kombination sprachlicher und materieller Bildern im Rahmen von Sprache-Bild-Texten heißt es bei Stöckl (2011: 48):

Sprache und Bild gehen im Gesamttext eine Arbeitsteilung der kommunikativen Funktion ein. Dabei gleichen die Stärken des einen semiotischen Systems die Schwächen des anderen aus und umgekehrt. So können Bilder merkmalsreiche Objekte vor Augen führen, Sprache aber kann konkrete Aspekte dieser Seherfahrung benennen und zu Handlungen explizit anleiten.

(Stöckl 2011: 48)

Eben diesen Doppelaspekt von Sprache-Bild-Texten nutzen Kollektivsymbole im oben definierten Sinn, indem sie sprachlich realisierte Bilder (Picturae mit den entsprechenden Potenzialen der Polyvalenz und Interpretationsoffenheit) mit den Möglichkeiten sprachlicher Sinnzuschreibungen auf der Ebene der Subscriptio kombinieren. Ähnliches lässt sich auch für den Gebrauch materieller Bilder feststellen, die in Form von Bildunterschriften, Artikelüberschriften und konkreten Erläuterungen im Rahmen der jeweiligen Begleitartikel von den Möglichkeiten der sprachlichen Interpretation und Monosemierung Gebrauch machen.

Ganz in diesem Sinne ist das Foto der Familie Wahafi in Abbildung 3 auf typische Weise mehrfach sprachlich-interpretatorisch eingebunden: Es ist integriert in einen Beitrag unter der Überschrift "Im Regen stehen gelassen". Damit verbindet bereits die Überschrift das Foto mit dem konkreten politischen Ereignis und positioniert sich zugunsten der Flüchtlinge. Die Bildunterschrift unter dem Foto ergänzt den analysierten Bildinhalt durch die Information: "Hanifeh Wahafi ist mit ihren beiden Kindern aus Afghanistan erst nach Iran geflohen." Diese spärlichen und verglichen mit der Überschrift irritierend neutralen Informationen, die vor allem den unbekannten Flüchtlingen einen Namen geben und ihre familiäre Zusammengehörigkeit bestätigen, sind prinzipiell dazu geeignet, den Beitrag auf weitere Details hin zu lesen. So erfahren Leser/innen im Beitrag tatsächlich mehr über die mehrjährige Flucht der Familie, ihr gewaltsames Auseinanderbrechen, den schlechten Gesundheitszustand der 28jährigen Mutter, der den Grund dafür bildet, dass die Familie noch nicht abgeschoben wurde.

Der Zusammenhang zwischen dem Foto und dem Beitragstext wird am deutlichsten mit den beiden den Beitrag abschließenden Sätzen kommentiert. Dort heißt es:

Die Mutter spricht Fasi, man verstünde sie nicht, würde nicht ein Dolmetscher aus dem Unterstützerkreis ins Englische übersetzen. Die Sprache der Augen aber versteht jeder, es ist die Sprache der Verzweifelten.

(Kastner 2013b) 
Während der übrige sprachliche Text die Bedeutung des Fotos durch weitere Detailinformationen zur Geschichte der Familie Wahafi anreichert, kann der letzte Satz eindeutig als Subscriptio zur fotografischen Darstellung gelesen werden, indem dem Foto ein konkreter Sinn zugesprochen wird.

Damit lässt sich im Anschluss an diese theoretischen Überlegungen aus semiotischer und diskursanalytischer Perspektive festhalten, dass materielle Bilder aufgrund ihrer spezifischen semiotischen Eigenschaften kognitive und emotionserzeugende Vorteile aufweisen, die bei Betrachter/inne/n anschauliche Vorstellungen hervorzurufen, persönlichere Beziehung zum konkreten Ereignis entstehen lassen und so potenziell Interesse und/oder Empathie wecken können. Jenseits dieser Potenziale haben Bilder jedoch den Nachteil, dass sie im Detail vage oder mehrdeutig bleiben und somit zwangsläufig der diskursiven Vereindeutigung bedürfen, sollen sie - mit Foucault (1977: 25) gesprochen - nicht im Vagen, im "Raum eines wilden Außen[s]" verharren. Die Tatsache, dass letzteres sowohl für materielle als auch für sprachliche Bilder gilt, erlaubt es, den Symbolbegriff, der zunächst für sprachliche Bildkomplexe entwickelt wurde (vgl. Link 1975), im Rahmen komplexer Sprache-Bild-Texte auch für materielle Bilder zu verwenden, wo immer diese (im Sinne einer Subscriptio) sprachlich näher bestimmt werden (können).

Hierauf aufbauend soll im folgenden Abschnitt am Beispiel von Teilereignissen des Hungerstreiks verdeutlicht werden, welche Bedeutung der Nutzung von materiellen und sprachlichen Bildern im Diskurs um den Hungerstreik zukam. Auch wenn man davon ausgehen kann, dass konkrete diskursive Ereignisse auf Seiten von Mediennutzer/inne/n als komplexe Kombination visueller und verbaler Elemente kognitiv verknüpft und gespeichert werden, so stellt sich natürlich dennoch die Frage, welche spezifischen Effekte mit der je konkreten semiotischen Realisierung verknüpft sind.

\section{Relevanz bildlicher Elemente am Beispiel unterschiedlicher diskursiver Positio- nen}

Während im Bisherigen die jeweiligen politischen Positionen der Frankfurter Allgemeinen und der Süddeutschen Zeitung zum Ereignis des Hungerstreiks am Rindermarkt nicht explizit kommentiert wurden, soll nun kurz auf die Unterschiede zwischen den beiden Zeitungen eingegangen werden, bevor im Weiteren die diskursiven Folgen der unterschiedlichen Nutzung von materiellen und verbalen Bildern aus semiotischer Sicht herausgearbeitet werden.

Zunächst einmal ist festzuhalten, dass der Umfang der Berichterstattung zum Hungerstreik in der Süddeutschen Zeitung den der Frankfurter Allgemeinen (sicher auch aus lokal-geografischen Gründen) deutlich überstieg. Während die Süddeutsche Zeitung in ihrer Onlineausgabe mit Beginn des Hungerstreiks täglich ein- bis zweimal berichtete, fanden sich in der Frankfurter Allgemeinen Zeitung erst gegen Ende des Streiks vereinzelte Hinweise auf das Ereignis. Darüber hinaus ließen sich sowohl in der Berichterstattung als auch in den Kommentaren signifikante Unterschiede hinsichtlich der expliziten Positionierung beider Zeitungen beobachten: Während die $S Z$ durchgängig auf die sachliche Angemessenheit der politischen Forderungen der Flüchtlinge hinwies und den Hungerstreik als "ultima ratio" politischer Verzweiflung interpretierte, standen im Mittelpunkt der Berichterstattung und Kommentierung der FAZ Hinweise auf die Unangemessenheit und Rechtswidrigkeit der eingesetzten Mittel. In- 
soweit kann jenseits der im Weiteren diskutierten Frage des Einsatzes von Bildern festgehalten werden, dass die diskursiven Positionen der beiden Zeitungen hinsichtlich des Hungerstreiks diametral entgegengesetzt waren. Im Hinblick auf das Rahmenthema dieses Heftes zum Zusammenhang zwischen Sprache und Politik ist die unterschiedliche diskursive Positionierung entscheidend für die Frage, ob bestimmte semiotische Strategien eine spezifische Präferenz zu einer der beiden vertretenen politischen Positionen haben.

Bevor jedoch eine solche diskurspraktische Auswertung der Ereignisse vorgenommen werden kann, geht es im diskursanalytischen Teil dieses Beitrags zunächst nicht um die politische oder ethische Kommentierung der beiden unterschiedlichen Positionen, ${ }^{4}$ sondern um die diskursiven Folgen der eingesetzten Bildelemente. Aus dieser Perspektive kann im Hinblick auf die Menge der gezeigten Fotos festhalten werden, dass sich in der Frankfurter Allgemeinen online zwei Fotos der Ereignisse fanden, während die Süddeutsche online täglich mit zwei bis drei bebilderten Berichten präsent war, die in der Regel je zwei Fotos enthielten.

Damit soll es im folgenden Teilkapitel unter Bezugnahme auf die vorhandenen materiellen Bilder des Streiks darum gehen aufzuzeigen, welche unterschiedlichen bildlichen Vorstellungen die beiden Zeitungen ihren Leser/inn/en vermittelten.

\subsection{Materielle Bilder und der Ereigniswert des Hungerstreiks}

Ausgehend von den vorhergehenden theoretischen Überlegungen ist im Hinblick auf die $F A Z$ entscheidend, dass die zwei während des Streiks (einen Tag vor Auflösung des Lagers) veröffentlichten Fotos in Abbildung 4 und 5 (FAZ) auf den ersten Blick keine explizite Stellungnahme nahe legen:

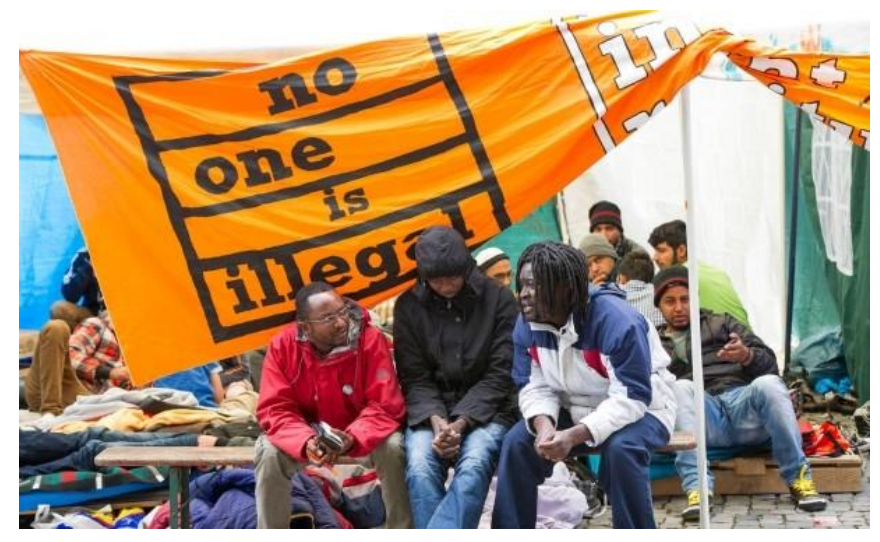

Abbildung 4: "Ein bitterböses Spiel mit Schicksalen"

Zwar zeigt das Foto in Abbildung 4 (FAZ online, 29.06.2013) vergleichbar mit dem oben gezeigten Bild der Familie Wahafi drei farbige Männer vor dem Hintergrund einer Gruppe weiterer Männer (innerhalb eines Zeltes), bei genauerem Hinsehen weisen die beiden Fotos jedoch deutliche Unterschiede auf: Keiner der drei frontal aufgenommen Männer in der $F A Z$ tritt in Blickkontakt mit den Betrachter/inne/n. Die Tatsache, dass sie ihren Blick abwenden, führt dazu, dass sie anders als Familie Wahafi nicht als Einzelne wahrgenommen werden, sondern als eine mit sich selbst beschäftige unbekannte Dreiergruppe. Letzteres wird dadurch

4 Für diesen Hinweis im Rahmen der Tagung "Sprache und Politik" möchte ich mich bei Thomas Niehr bedanken. 
unterstrichen, dass es anders als in Abbildung 3 keine Schärfeunterschiede zwischen dem Vordergrund ("Dreiergruppe") und dem Hintergrund ("Gruppe der übrigen Flüchtlinge") gibt.

Darüber hinaus weckt das insgesamt bunt wirkende Foto anders als das in Abbildung 3 betrachtete keine Assoziationen einer hoffnungslosen Situation. Stattdessen wirkt die abgebildete Szene unpersönlich und politisch abstrakt, was zusätzlich durch die große orange Banderole mit der Aufschrift "no one is illegal" unterstrichen wird, deren (innerhalb dieses Fotos unkonkrete) Botschaft ebenfalls keine spezifischen politischen Forderungen erkennen lässt. Ein dritter Unterschied besteht darin, dass im Gegensatz zum analysierten Foto einer Mutter mit ihren Kindern im vorliegenden Fall alle abgebildeten Flüchtlinge Männer sind, was im Hinblick auf die Evokation von Angst potenziell einen entscheidenden Unterschied darstellt. ${ }^{5}$

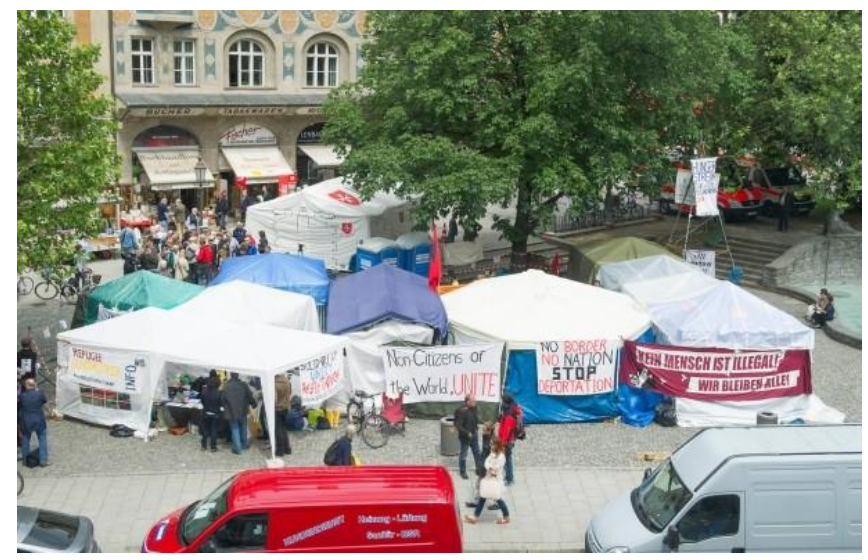

Abbildung 5: "Ein bitterböses Spiel mit Schicksalen"

Auch das zweite Foto aus der Frankfurter Allgemeinen (FAZ online, 29.06.2013) in Abbildung 5 bleibt hinsichtlich seines politischen und emotionalen Gehalts unscharf: Betrachter/innen sehen aus der Vogelperspektive Zelte und Menschen auf einem Platz, jedoch enthält auch diese Szene keine spezifischeren individuellen oder persönlichen Informationen. Die erkennbaren Banderolen lassen politische Kontexte vermuten. Genaueres ist aufgrund der Perspektive und der Entfernung der Kamera jedoch nicht zu erkennen. Zusätzlich lässt sich festhalten, dass die Perspektive der Aufnahme (von oben auf die Zelte) auf ein hierarchisches Gefälle zwischen Betrachter/in und Ereignis verweist.

Damit sprechen beide Fotos auf der Darstellungsebene dem Ereignis des Hungerstreiks keinen prägnanten politischen Nachrichtenwert zu. Es handelt sich um "irgendeinen" Protest "irgendwelcher" Menschen, die selbst jedoch nicht als politisch handelnde Akteure in den Blick kommen. Darüber hinaus legen sowohl der fehlende Blickkontakt auf dem ersten Foto als auch die Aufnahme aus der Vogelperspektive auf dem zweiten Bild Betrachter/inne/n insgesamt eine tendenziell negative Perspektive nahe.

Wird nun im nächsten Schritt zusätzlich berücksichtigt, anhand welcher konkreten Subscriptiones die bildliche Botschaft in den Gesamttext des Berichts integriert wird, so fällt im Zusammenhang mit der Überschrift "Ein bitterböses Spiel mit Schicksalen" (FAZ online, 29.06.2013) auf, dass auch diese eine negative Wertung enthält, wobei zunächst unklar bleibt,

5 Schaut man sich die bildliche Berichterstattung zu Flüchtlingen an, so lässt sich festhalten, dass Flüchtlinge häufig entweder als "geschlechtsindifferente Massen" oder als "Männer" Angst auszulösen scheinen. 
wer eigentlich ein "bitterböses Spiel" betreibt. Erst im Fließtext des Berichts (FAZ online, 29.06.2013) werden die bestehenden inhaltlichen Leerstellen schrittweise konkretisiert: So werden die Flüchtlinge zu Beginn des Beitrags als 'Störung' (Subscriptio) eines "sonnigen Freitag(s) am Münchener Rindermarkt" (Pictura) sprach-bildlich inszeniert. 'Kontakt' mit ihnen sei nicht möglich (Subscriptio), "da sie sich eine kleine, abgeschlossene Zeltstadt gebaut" hätten (Pictura). Als das Problem der Flüchtlinge wird nicht ihre politische Lage begriffen, sondern die Tatsache, dass "sie ihre Gesundheit aufs Spiel" setzen (Subscriptio). Die Verantwortung hierfür wird dem Sprecher der Gruppe (siehe oben "der Mann") angelastet, der auch hier erneut nicht namentlich genannt wird, und den Unterstützern der Flüchtlinge, "die sich in einer kruden Kapitalismuskritik gefallen". Beiden Protagonisten werden "politische Geschäfte unter dem Vorwand humanitärer Unterstützung" und damit eben ein "bitterböse[s] Spiel" vorgehalten. Die Flüchtlinge selbst werden hierbei nicht als selbständig handelnde Akteure dargestellt, sondern in doppeltem Sinne negativ als unselbständige Handlanger und Opfer innerhalb dieses "bitterböse[n] Spiel[s]" von Dritten bestimmt.

Dieser Entmündigung der Flüchtlinge bei gleichzeitiger negativer Bewertung des Hungerstreiks als "bitterböses", potenziell 'tödliches' Spiel wird ein weiteres sprachliches Bild in Form eines Symbols gegenübergestellt, wenn es heißt:

Es ist nicht zu erfahren, ob sie (die Hungerstreikenden; D. M.) wahrnehmen, welche Anstrengungen unternommen werden, damit sie keinen Schaden nehmen; Krankenwagen stehen am Rindermarkt bereit, sie zur ärztlichen Versorgung in Kliniken zu fahren.

(Schäffer 2013a)

Das Symbol des Krankenwagens, das hier explizit anhand der Subscriptiones "beschützend", "fürsorglich", "behördlich" und "deutsch" semantisch präzisiert wird, wird im folgenden Abschnitt dieses Beitrags erneut thematisiert (vgl. Kapitel 3.2). Bereits an dieser Stelle sei jedoch darauf hingewiesen, dass das Symbol des Krankenwagens dazu dient, dem unangemessenen Verhalten der Flüchtlinge das verantwortungsvolle Verhalten deutscher Behörden entgegenzusetzen.

Dieser Kontrast wird unter Bezug auf eine Erklärung der Hungerstreikenden, die deren Affinität zu (ebenfalls hungernden) Terroristen wie Bobby Sands und Holger Meins andeutet, unterstrichen, indem der (vermeintlich) terroristische Hintergrund der Flüchtlinge explizit benannt wird:

Deutlicher als mit dem Verweis auf das IRA-Mitglied Bobby Sands [...] und zu dem RAFTerroristen Holger Meins, die sich zu Tode hungerten, kann nicht werden, welche politischen Geschäfte unter dem Vorwand humanitärer Unterstützung getrieben werden.

(Schäffer 2013a)

Schaut man sich an dieser Stelle exemplarisch das Zusammenspiel materiell-bildlicher und symbolisch-sprachlicher Strategien an, so lässt sich festhalten, dass die emotionale Wirkung der materiellen Bilder in der Frankfurter Allgemeinen nicht vorrangig auf der Ebene der Darstellung entsteht, sondern vielmehr dadurch, dass auf den Ebenen der Interaktion und der Vertextung der Fotos eine negative Bewertung der dargestellten Situation nahe gelegt wird. Der konkrete Gehalt dieser Wertung bleibt jedoch materiell-bildlich im Dunklen. Gerade diese fehlende Prägnanz lässt das hieran anschließende symbolische Spiel mit sprachlichen Bildern in Form von Negativklischees umso effektiver verlaufen. 
Inwieweit es sich bei der beobachteten Verbindung aus inhaltlicher Vagheit und negativen Wertungen bezogen auf den Einsatz materieller Bildern um ein systematisches Potenzial journalistischer Fotoberichterstattung handelt, kann hier nicht entschieden werden. Die Empirie des vorliegenden Beispiels unterstreicht eine solche Annahme allerdings insoweit, als dass die semiotischen Strategien der Süddeutschen Zeitung im Gegensatz zur Frankfurter Allgemeinen Zeitung an allen genannten Punkten als konträr beschrieben werden müssen.

So werden im Rahmen der Berichterstattung der $S Z$, ähnlich wie oben bereits anhand des Fotos der Familie Wahafi gesehen, immer wieder konkrete Bilder von den Protagonist/inn/en des Hungerstreiks vor oder in den Zelten am Rindermarkt gezeigt. Anders als in Abbildung 4 (FAZ) sehen die in Abbildung 6 gezeigten Flüchtlinge (SZ, 16:32 Uhr) die Betrachter/innen direkt an. Damit nehmen sie nicht nur als Protagonisten des Hungerstreiks durch den direkten Blick in die Kamera auf interaktiver Ebene Kontakt zu Leser/inne/n auf und werden als handelnde Akteure sichtbar, sondern auch der Hungerstreik wird menschlich real.

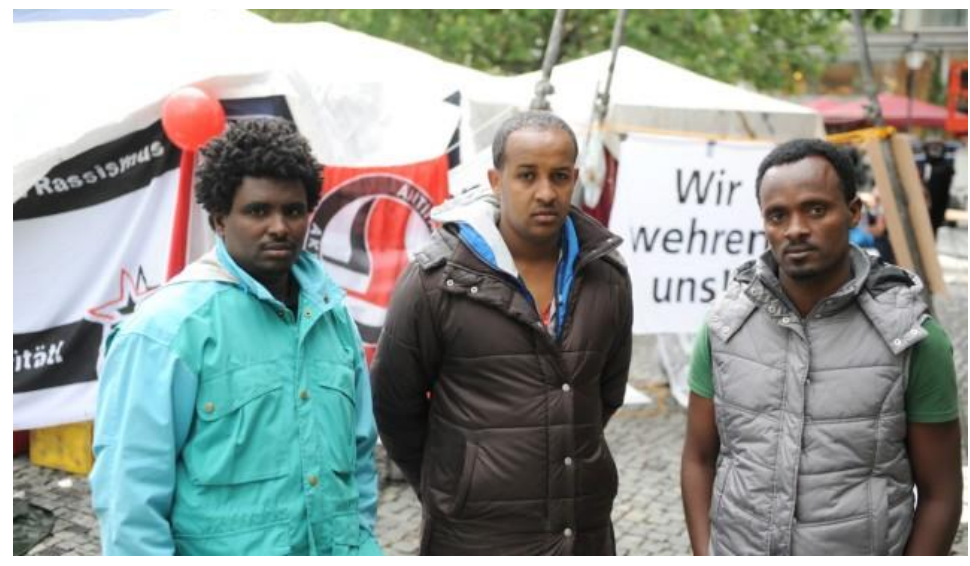

Abbildung 6: "Menschen zweiter Klasse"

Gleichzeitig bietet auch der sprachliche Text, in den das vorliegende Foto integriert ist, eine Perspektive der menschlichen Betroffenheit an, die schon anhand der Überschrift "Menschen zweiter Klasse" deutlich wird. So heißt es im Bericht:

Wer die Hungerstreikenden besucht, blickt in traurige, aber entschlossene Gesichter. Viele von ihnen sind traumatisiert und haben in ihrer Heimat und auf der Flucht erlebt, was kaum jemand in diesem Land sich vorstellen kann. Sie stehen stellvertretend für Tausende. Als Bürger dieses Staates schämt man sich, dass eben dieser Staat Hilfesuchende systematisch wie Menschen zweiter Klasse behandelt.

(Kastner 2013c)

Damit werden auf der sprachlich-symbolischen Ebene Subscriptiones wie 'Trauer', 'Entschlossenheit' und 'Traumatisierung' als menschliche Eigenschaften der Flüchtlinge aufgerufen. Auch hier wird die Abbildung der Flüchtlinge damit durch weitere Informationen ergänzt, die ihnen den Status eigenständiger Individuen zusprechen.

Noch einen Schritt weiter geht das Foto in Abbildung 7 (SZ), indem es ebenfalls konkrete Akteure des Streiks, die in die Kamera sehen, zeigt, diesen Aspekt aber durch eine vor den Flüchtlingen positionierte Banderole zusätzlich an konkrete politische Botschaften knüpft, aus denen sowohl die Widerständigkeit der Hungerstreikenden als aber auch ihre Forderung des Bleiberechts hervorgeht. Diese Kopplung konkreter Einzelfälle an politische Forderungen trägt dazu bei, dass der Streik auf bildlicher Ebene zu einem realen politischen Ereignis wird, 
das bei Betrachter/inne/n Interesse und potenziell emotionale Reaktionen wie Mitgefühl auslöst.

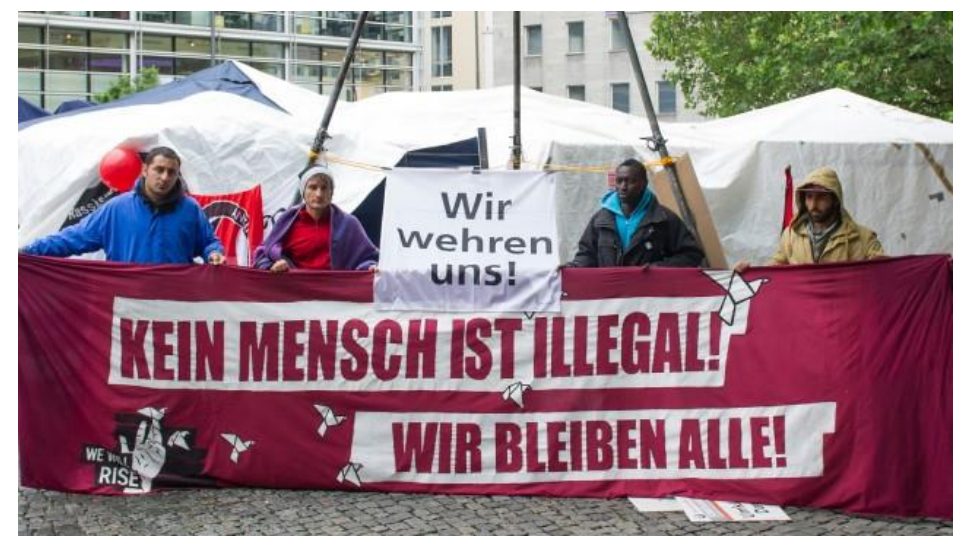

Abbildung 7: "Heimatlos an der Isar"

Letzteres wird durch den sprachlichen Text des Beitrags dadurch unterstrichen, dass unter der Zwischenüberschrift "Mich berührt das persönlich" eine Münchener Bürgerin beschrieben wird:

\begin{abstract}
"Mich berührt das persönlich, das Schicksal dieser Menschen", sagt Frau Gerber und erzählt von ihrem Sohn, der als Arzt neulich in Syrien geholfen hat, der Schlimmes gesehen hat in einem Land, aus dem die Menschen auch fliehen müssen. "Wir sind ein so unheimlich reiches Land!", ruft Frau Gerber in die Junikälte, weil sie nicht versteht, dass die, die es bis zu uns geschafft haben, so schäbig behandelt werden. Sie kennt ja auch die Fotos von den Jugendlichen, die auf einem alten Münchner Militärareal untergebracht sind, der "Bayernkaserne". Minderjährige, die ohne Eltern gekommen sind, "sie haben ja keine Zukunft".
\end{abstract}

(Kastner 2013d)

Die Kopplung zwischen den Einzelschicksalen und dem politischen Nachrichtenwert des Hungerstreiks wird in der SZ also sowohl auf materiell-bildlicher als auch auf sprachlicher Ebene realisiert, wodurch unter Nutzung beider semiotischen Modalitäten diffuse emotionale Betroffenheit zu parteilicher Empathie präzisiert wird.

Im Hinblick auf die Analyse materieller Bilder lässt sich zusammenfassend festhalten, dass die in der $F A Z$ veröffentlichten Fotos trotz einer deutlich negativen Bewertungstendenz im Detail der dargestellten Inhalte vage bleiben, während die $S Z$ die Hungerstreikenden und ihr Anliegen bildlich explizit und positiv in den Mittelpunkt stellt. Am deutlichsten werden die Unterschiede zwischen den Bildstrategien beider Zeitungen auf der Interaktionsebene: Hier setzt die $S Z$ darauf, eine Beziehung zwischen den Protagonist/inn/en des Ereignisses und den Betrachter/inne/n entstehen zu lassen, wohingegen die $F A Z$ den Betrachter/inne/n einen Beobachterstatus aus der Distanz anbietet, wodurch die im Hungerstreik handelnden Protagonist/inn/en unpersönlich und abstrakt bleiben. Diese unterschiedlichen Zugänge zu den Fotos können dann im Rahmen des sprachlichen Textes für Sinnangebote genutzt werden, die den Bildern im Detail eine eindeutig negativ-ablehnende $(F A Z)$ bzw. positiv-unterstützende Bedeutung $(S Z)$ zuordnen. Insoweit bieten beide Zeitungen auf der sprachlichen Ebene Subscriptiones zu den gezeigten Fotos an, die die in den Fotografien enthaltenen wertenden Tendenzen monoseminieren und dadurch die Fotos zusätzlich semantisch aufladen. 
Bezogen auf das vorliegende Material lässt sich bereits an dieser Stelle festhalten, dass sich diese Art der Nutzung materiell bildlicher Elemente in beiden Zeitungen als entscheidend für die Bestimmung der diskursiven Relevanz der Ereignisse am Rindermarkt erweist. So können die bisherigen empirischen Befunde aus semiotischer Sicht im Anschluss an Meier (2005: 129) sowohl mit dem ikonischen Charakter fotographischer Bildzeichen erklärt werden, die die Anschaulichkeit der Ereignisse erhöhen, als auch mit ihrer indexikalischen Qualität, die die Tatsache unterstreicht, dass es überhaupt ein Ereignis gibt. Auf letzteres aufbauend verweist auch die unterschiedliche Menge der veröffentlichten Fotos in FAZ und $S Z$ auf eine unterschiedliche diskursive Positionierung beider Zeitungen.

Darüber hinaus ist unter Bezug auf die relative Vagheit bzw. Mehrdeutigkeit von Bildzeichen deutlich geworden, dass die Existenz von materiellen Bildern alleine keine eindeutigen Aussagen hinsichtlich ihrer Funktion im Diskurs zulässt. Vielmehr haben die Beobachtungen gezeigt, dass materielle Bilder zwar Anschlussoptionen für ihre Interpretation eröffnen, sie ihre konkrete diskursive Bedeutung jedoch erst im textuellen Gesamtzusammenhang entwickeln. Damit soll im nächsten Abschnitt die unterschiedliche Nutzung sprachlicher Bilder im Rahmen des Hungerstreiks genauer betrachtet werden.

\subsection{Diskursive Kämpfe um die Interpretation sprachlicher Bilder während des Hungerstreiks}

Im Zusammenhang mit dem gesellschaftlichen und politischen Nutzen von Kollektivsymbolen stellt Link (1983: 11, 1985: 68) in unterschiedlichen Kontexten heraus, dass gerade die relative Vagheit der Bildseite von Symbolen (der Pictura) einen der Gründe dafür bildet, dass Kollektivsymbole als Mittel der Auseinandersetzung besonders gut geeignet sind. Die Relevanz dieser Annahme soll in diesem Abschnitt anhand des Einsatzes sprachlicher Bilder während des Hungerstreiks verdeutlicht werden.

Konkret geht es im folgenden Beispiel um eine schwangere Frau, die im Rahmen der Berichterstattung der Süddeutschen kurzfristig zum Symbol der politischen Auseinandersetzung wurde. Zum ersten Mal erwähnt wird die Schwangere in der $S Z$ am 4. Tag des Streiks von einer Sozialreferentin der Stadt München, Brigitte Meier, die sich der $S Z$ folgend mit den Hungerstreikenden solidarisiert hatte. Die Süddeutsche Zeitung schreibt:

"Ihre Forderungen (die der hungerstreikenden Flüchtlinge; D. M.) sind berechtigt", erklärt Meier, "wir verstehen sie". Das sagt sie auch in Richtung Staatsregierung, die für die Unterbringung und Behandlung der Flüchtlinge zuständig ist. Meier hat unter den Planen eine hochschwangere Frau gesehen. Wenn diese Frau lieber im Regen auf dem Rindermarkt ausharre als in ihre Unterkunft zu gehen, sagt Meier, dann sollte die Staatsregierung doch mal in sich gehen.

(s. n. 2013a)

Die Subscriptio, die Meier zur Pictura der hochschwangeren Frau "unter den Planen" anbietet, ist die der 'extremen Notlage'. Meier interpretiert die Anwesenheit einer Frau kurz vor der Entbindung unter den Hungerstreikenden somit als Beleg für das Ausmaß der Not der werdenden Mutter.

Einen Tag später findet sich in der Süddeutschen folgendes Zitat der bayerischen Sozialministerin Christine Harderthauer: 
Dass eine schwangere Frau in den Hungerstreik geht und damit ihr Ungeborenes gefährdet, muss sofort beendet werden, hier sollten sich alle einig sein.

(s. n. 2013b)

Während Meier bei ihrer Interpretation des Symbols der "Schwangeren" deren Verantwortung für ihr (ungeborenes) Kind als konstitutiv voraussetzt, verschiebt sich die Perspektive im Zitat Harderthauers genau entgegengesetzt auf die Subscriptio der 'Verantwortungslosigkeit der Mutter'. Gleichzeitig kündigt Harderthauer in Anbetracht der behaupteten Schädigung des ungeborenen Kindes denkbare Interventionen des bayerischen Staates an, den sie in der (symbolischen) Position des lebensrettenden Helfers sieht (siehe dazu das in 3.1 bereits angesprochene Symbol des Krankenwagens).

Aus semiotischer Sicht deutet das vorhergehende Beispiel damit zunächst einmal darauf hin, dass die semantische Besetzung sprachlicher Bilder (Picturae) aufgrund der Explizitheit sprachlicher Zeichen bereits im Ansatz und damit ungleich eindeutiger erfolgt, als dies bei Fotos der Fall ist. Gleichzeitig ist mit dieser Feststellung jedoch die Tatsache verknüpft, dass dem propositionalen Gehalt der Subscriptio auch ungleich leichter widersprochen werden kann, indem dem genutzten sprachlichen Bild ein anderer - im vorliegenden Fall - genau entgegengesetzter Sinn zugeordnet wird.

Dass gerade die Kombination aus (sprachlichen) Bildelementen und diesen zugeordneten Sinneinheiten in besonderem Maß zur flexiblen Nutzung sprachlicher Symbole beiträgt, wurde im Rahmen des Hungerstreiks daran deutlich, dass das Symbol der Schwangeren in der Süddeutschen zwar nur kurze Zeit diskursiv präsent war, die mit diesem Symbol verbundene Subscriptio der 'systematischen Selbst- und Fremdschädigung' der Flüchtlinge jedoch in der Berichterstattung der FAZ kontinuierlich weiter genutzt wurde: So berichtet die Frankfurter Allgemeine (01.07.2013: 3) am Morgen nach der polizeilichen Auflösung des Hungerstreiks beispielsweise darüber, dass es "drei Kinder" unter den Hungerstreikenden gegeben habe (Subscriptio: 'Missbrauch von Schutzbefohlenen') und dass die während des Hungerstreiks zusammengebrochenen und in Krankenhäusern behandelten Flüchtlinge danach zu den Hungerstreikenden zurückgekehrt seien (Subscriptio: 'Selbstschädigung').

In der Folge interpretiert die $F A Z$ die Auflösung des Lagers am 30.06.2013 durch ein "Großaufgebot der Polizei" und durch "Rettungskräfte" in ihrem Kommentar wie folgt:

Bekannt ist [...], dass sie (die Hungerstreikenden; D. M.) sich in die Hände linksextremer Unterstützer begeben haben, die kaltblütig die Gefährdung ihrer "Schützlinge" in Kauf nahmen, um ihre eigene antikapitalistische Kampagne zu fahren. Diesen Leuten hat das Wohlergehen der Asylbewerber offensichtlich zuallerletzt am Herzen gelegen. Andererseits hätten sie das Entgegenkommen staatlicher Stellen, die Asylanträge beschleunigt zu prüfen, nicht für das propagandistische Ausschlachten des auch von ihnen zu verantwortenden Leidens der Menschen schnöde abgelehnt. Vor dieser tödlich gefährlichen Instrumentalisierung hat die Polizei die Asylbewerber vorerst gerettet.

(Wagner 2013)

Die Oppositionsbildung zwischen den Polen der Schädigung der Flüchtlinge durch "linksextreme Unterstützer" und der Rettung der Flüchtlinge "durch die Polizei" wird unterstützt durch die Semzuschreibungen "radikal", "herzlos", "kaltblütig" und "berechnend" auf der einen und die Seme "staatlich", "entgegenkommend" und "rettend" auf der anderen Seite. Die Flüchtlinge selber erscheinen auch hier nicht als handelnde Personen. 
Während die genannten semantischen Zuschreibungen im zitierten Auszug vorrangig als Subscriptiones realisiert werden, findet sich am gleichen Tag eine Bildstrecke von 4 Fotos in der FAZ online, die die genannten Seme bildlich aufgreift:

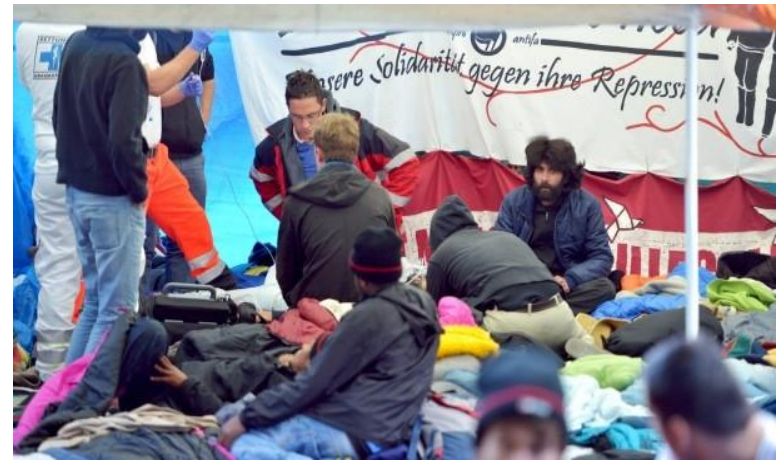

Abbildung 8: "Hungerstreik: Münchner Flüchtlingsprotest nach Großeinsatz beendet"

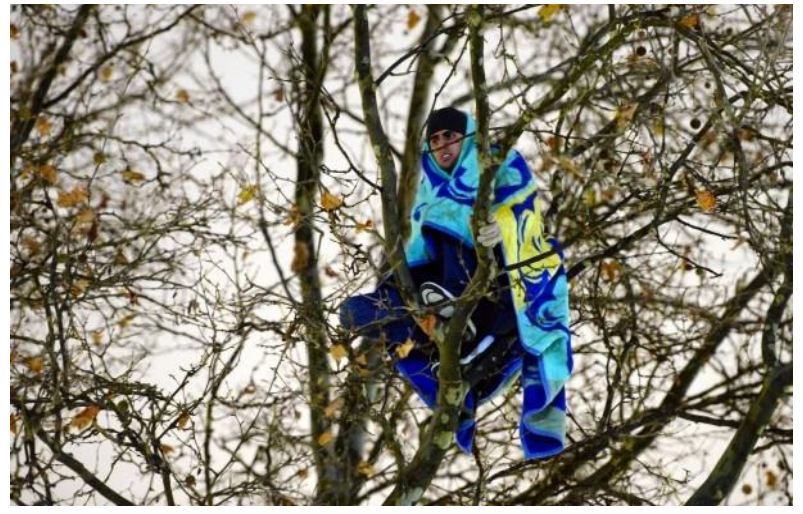

Abbildung 9: "Hungerstreik: Münchner Flüchtlingsprotest nach Großeinsatz beendet"

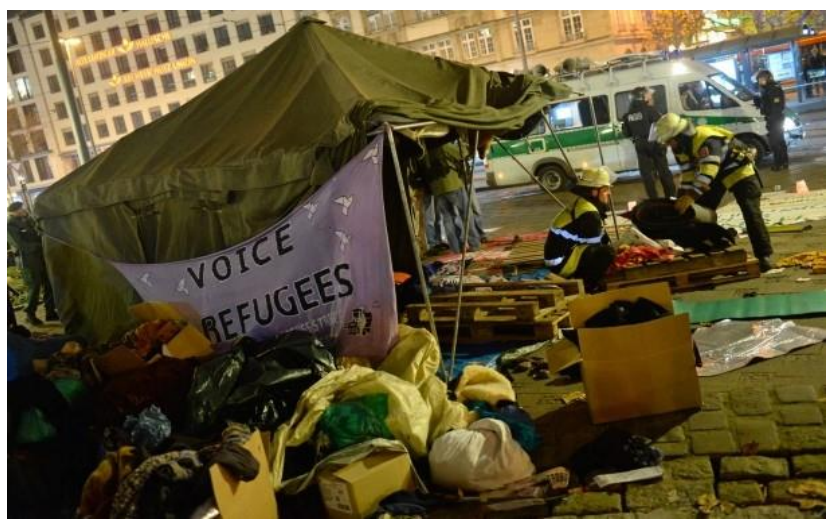

Abbildung 10: "Hungerstreik: Münchner Flüchtlingsprotest nach Großeinsatz beendet"

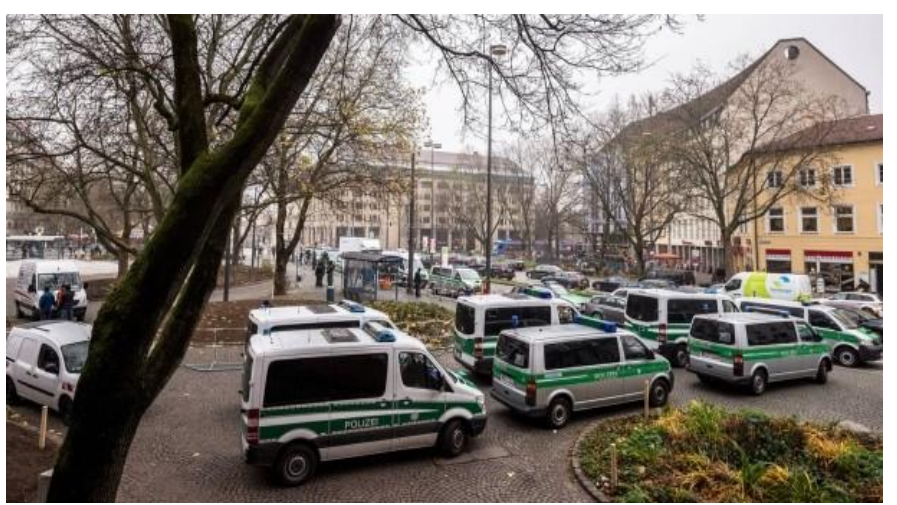

Abbildung 11: "Hungerstreik: Münchner Flüchtlingsprotest nach Großeinsatz beendet" 
Ohne an dieser Stelle im Detail auf die vier Fotos eingehen zu können, lassen sie sich in Teilen als analoge bildliche Nacherzählung des wiedergegebenen Zitats interpretieren: So zeigt das Foto in Abbildung 8 sowohl einen langhaarigen, bärtigen Mann (Subscriptio: 'linksextreme Unterstützer'), als auch Helfer des THWs (Subscriptio: 'Entgegenkommen staatlicher Stellen'). Der Flüchtling in Abbildung 9, der vor der Polizei auf einen Baum geflohen ist (Bildunterschrift in der FAZ online vom 01.07.2013), wird im Rahmen der fotografischen Darstellung zum hilflosen Opfer des Hungerstreiks. Abbildung 10 zeigt das aufgelöste Lager, das jetzt von staatlichen Helfern und Polizisten abgeräumt wird (Subscriptio: 'Sieg der Rechtstaatlichkeit'), was unter Einbezug von Abbildung 11 als das Ergebnis der rechtsstaatlichen Übermacht interpretiert werden kann.

Damit erweisen sich die Bilder der Fotostrecke einerseits als belegende Illustrationen der Berichterstattung, indem sie sowohl indexikalisch wie ikonisch auf die Räumung des Lagers verweisen, andererseits eröffnen sie aufgrund ihrer Polyvalenz semantische Optionen, die sprachlich kaum realisierbar gewesen wären. Letzteres gilt vor allem für Abbildungen 9 und 11: Weder die Analogie zwischen einem Flüchtling auf einem Baum und einem Affen, noch die Botschaft des Sieges einer polizeilichen Übermacht könnte in dieser Form von einer seriösen deutschen Zeitung explizit sprachlich realisiert werden.

Im Anschluss an die beschriebene Gegenüberstellung der bayerischen Behörden auf der einen und den Unterstützern des Hungerstreiks auf der anderen Seite soll nun im Anschluss an die negative Wertungen des Hungerstreiks in der FAZ in einem letzten Schritt auf die Nutzung bildlicher Elemente für die Konstruktion von Feindbildern in der Form absoluter Negativsymbole (vgl. Link 1982: 13) eingegangen werden. Als Beispiel für ein solches Negativsymbol soll die diskursive und bildliche Darstellung des bereits mehrfach erwähnten "Manns" und "selbsternannten Führers", Ashkan Khorasani, betrachtet werden.

\subsection{Zur bildlichen Konstitution von Feindbildern}

Während in Abschnitt 3.1 deutlich wurde, dass es im Hinblick auf die direkte und positive Ansprache von Zeitungsleser/inne/n notwendig ist, sie mit bebilderten Einzelfallberichten anzusprechen und unpersönliche und abstrakte Bilder potenziell die Grundlage von Negativwertungen bilden, soll es nun um die Funktion von Bildelementen im Zusammenhang mit der Konstruktion absoluter Feindbilder am Beispiel des Symbols des "Fanatikers" und des "Terroristen" gehen.

In diesem Zusammenhang hat sich im letzten Abschnitt bereits die Strategie der Frankfurter Allgemeinen (vgl. FAZ, 01.07.2013) angedeutet, die Gruppe der Flüchtlinge immer wieder als "verführte" oder willenlose Opfer "linksextremer Unterstützer" zu inszenieren. Ebenfalls mehrfach wurde ein "Mann" und "selbsternannter Führer" erwähnt, Ashkan Khorasani, der jedoch auch in der $S Z$ erst einen Tag vor der Auflösung des Lagers der Hungerstreikenden namentlich genannt wird, und der in der $F A Z$ sogar erst nach der Auflösung des Lagers erwähnt wird.

So veröffentlicht die $S Z$ einen Tag nach der Auflösung des Lagers im Lokalteil eine einspaltige Charakteristik Khorasanis, in der er als "sympathisch", "charismatisch", 'wortstark' und 'gebildet', "unerbittlich" (Zitat Ude) und "radikalisiert", aber "gewaltfrei" beschrieben wird. 
Unter dieser Charakteristik findet sich das in Abbildung 12 (SZ, 01.07.2013) wiedergegebene Foto von Khorasani.

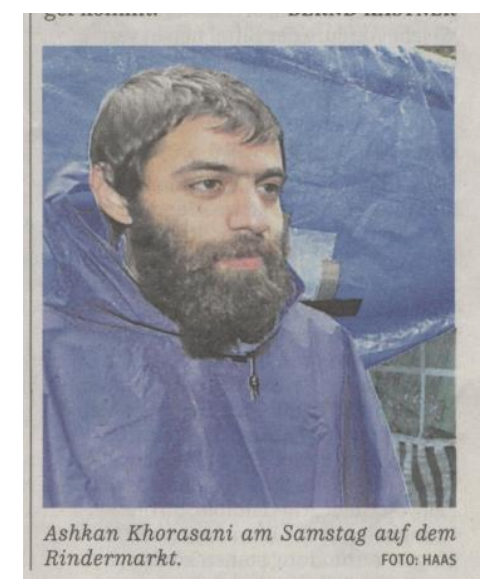

Abbildung 12: "Charismatisch und unerbittlich"

Ausgehend von dieser Charakteristik kann man vermuten, dass es u. a. der Nationalität und der Ambivalenz Khorasanis geschuldet war, dass die $S Z$ zu keinem Zeitpunkt versucht hat, ihn als Sprecher der Flüchtlinge positiv aufzubauen. Das vorliegende Foto in Abbildung 12 unterstreicht diese Annahme zumindest insoweit, als dass Khorasani die Betrachter/innen nicht ansieht und damit auf der Interaktionsebenebene keine Kontaktangebote macht.

Die diskursive Strategie der FAZ einen Monat nach der Auflösung des Lagers geht deutlich darüber hinaus. So veröffentlicht die Frankfurter Allgemeine (31.07.2013), also vier Wochen nach dem Ende des Hungerstreiks einen längeren Bericht mit einem Foto von Khorasani und zwei weiteren Iranern. Der Bericht wird wie folgt eingeleitet:

Als Hans-Jochen Vogel im Juni das Zeltlager von hungerstreikenden Asylbewerbern am Münchener Rindermarkt besuchte, kam ihm schnell der Verdacht, es mit "Fanatikern" (Vogel) zu tun zu haben. Der frühere SPD-Vorsitzende war von Bayerns Ministerpräsident Horst Seehofer (CSU) und Münchens Oberbürgermeister Christian Ude (SPD) gebeten worden, gemeinsam mit dem früheren Landtagspräsidenten Alois Glück (CSU) als Vermittler die wachsende Lebensgefahr für die Hungerstreikenden abzuwenden. Vor beiden stand nun, mit Regenmantel und Vollbart, Ashkan Khorasani, ein aus Iran geflüchteter Kommunist, der sich als Bote der hungerstreikenden Flüchtlinge vorstellte.

(Bender 2013)

Die expliziten und semantischen Zuschreibungen, die hier vorgenommen werden, lassen sich mit "fanatisch", "iranisch", "kommunistisch" und "bärtig" auf der Seite Khorasanis und als "vermittelnd" und "lebensrettend" auf Seiten der politischen Vertreter der bayerischen Regierung fassen. 


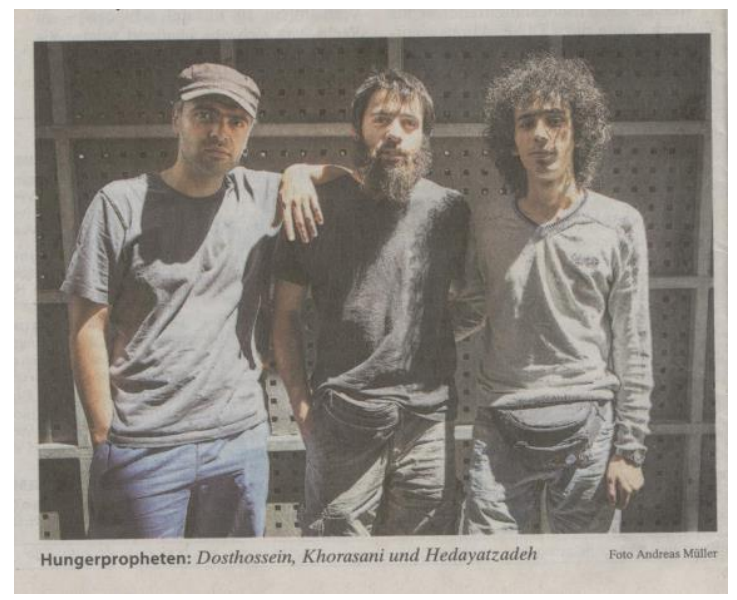

Abbildung 13: "Hungern, bis der Antrag durch ist"

Die sich hier andeutende Gegenüberstellung einer Koalition aus "Hungerpropheten", wie das in Abbildung 13 gezeigte Foto aus der $F A Z$ vom 31.07.2013 unterschrieben ist, und einer parteiübergreifenden "großen Koalition deutscher Vernunft" weist deutliche Merkmale von Feinbildstereotypen auf: Es findet sich die bipolare Gegenüberstellung der Akteure entsprechend der Pole "gut" vs. "böse", die semantischen Zuschreibungen Khorasanis (und eines seiner Kollegen, der dritte Iraner wird im Artikel gar nicht erwähnt) erfolgt anhand absoluter Negativstereotypen (als "iranische Fanatiker", "Kommunisten" und "Hungerprophet"), und das Foto in Abbildung 13 unterstreicht diese semantischen Zuschreibungen zumindest in Teilen durch die ethnische Herkunft der drei Männer, sowie den Bart Khorasanis und die langen schwarzen Haare des Iraners auf der rechten Seite.

Obgleich die Abbildung der drei Männer in Abbildung 13 jedoch einige Negativmerkmale enthält (siehe dazu auch den Kontrast zwischen den Fotos von Khorasani in den Abbildungen 12 und 13), muss man sich dennoch die Frage stellen, ob dieses Foto insgesamt die Anforderungen eines absoluten Negativklischees erfüllt. Dagegen spricht sowohl die freundschaftliche Umarmung der drei Männer als auch die Tatsache, dass ihre Blicke den Betrachter/inne/n offen begegnen. Hier ist zu vermuten, dass der Mangel an anderen Fotos den Grund für dieses Bild darstellte.

Den Auslöser dafür, dass die FAZ einen Monat nach dem Ende des Hungerstreiks mit dem vorliegenden Bericht reagierte, bildete vermutlich die Ankündigung weiterer Protestmärsche von Flüchtlingen durch Bayern ( $F A Z, 31.07 .2013: 3)$. Hätten diese eine ähnliche Aufmerksamkeitswelle ausgelöst wie der Hungerstreik am Rindermarkt, so hätte sowohl der Bericht als auch das Foto eine Grundlage bilden können, um weitere eindeutigere bildliche Negativzuschreibungen Khorasanis zu prozessieren. Bezogen auf den vorliegenden Zusammenhang bestätigt dieser erste Versuch des Aufbaus eines Negativsymbols "Khorasani" aus diskurssemiotischer Sicht in jedem Fall die Annahme, dass langfristig sowohl positive wie negative Bewertungen politischer Ereignisse nicht ohne materiell-bildliche Realisierungen auskommen. 


\subsection{Diskurssemiotische Auswertung}

Die bisherigen Beobachtungen haben aus unterschiedlichen Perspektiven gezeigt, dass Bilder - egal ob sprachliche oder materielle - hinsichtlich ihrer Wirkungen im Rezeptionsprozess politisch in der Regel vage, vielfach aber auch ambivalente Potenziale enthalten. Dies hat seinen Grund in ihrer (verglichen mit nicht-bildlichen Zeichen) intentionalen Offenheit, die aus semantischer und politisch Sicht "Risiken" nach sich zieht, die es diskursiv zu bändigen gilt. Diese Funktion der Deutung von Bildern übernehmen - wie anhand des hier untersuchten Materials gesehen - im Rahmen konkreter Sprache-Bild-Texte sprachlich realisierte Subscriptiones.

Trotz dieser Gemeinsamkeiten zwischen materiellen und sprachlichen Bildern haben die exemplarischen Beobachtungen dieses Beitrags verdeutlicht, dass sich die Wirkung von Bildern in Abhängigkeit von den jeweils genutzten semiotischen Ressourcen unterscheiden. So übersteigen die assoziativen und emotionserzeugenden Potenziale materieller Bilder deutlich die Möglichkeiten der sprachlichen Realisierung von Bildelementen. Gerade dieses emotionale Potenzial materieller Bilder hat sich aus empirischer Sicht als bedeutsam für politische $\mathrm{Zu}$ sammenhänge erwiesen: Materielle Bilder können sowohl Sympathie und Empathie erzeugen, als auch negative Wertungen hervorrufen, ohne die eine oder die andere emotionale Wertung direkt präzisieren zu müssen. Damit erweisen sie sich als ideale Anschlussstellen sowohl für die Möglichkeiten der politischen Solidarisierung als aber auch für die Konstruktion von Feindbildern.

Insgesamt ist damit eine Teilautonomie materieller Bilder deutlich geworden, die darin besteht, dass Bilder es erlauben, schnell und unhinterfragt eine je nach Arrangement unterschiedliche und eigenständig interaktive Beziehung zum Betrachter aufzubauen. In diesem Zusammenhang können materielle Bilder Assoziationen nahe legen, die diskursiv explizit kaum oder auch gar nicht benennbar sind. Diese Tatsache wird dadurch unterstützt, dass materiellen Bildern auf Grund ihrer unscharfen Proposition nur schwer widersprochen werden kann, was ebenfalls ein Grund für ihre Wirkungsmächtigkeit ist.

Im Gegensatz dazu wird die Vagheit bildlicher Eindrücke im Rahmen der Nutzung sprachlicher Bilder deutlich schneller relativiert, indem die Mehrdeutigkeit und Ambivalenz von Picturae durch die sprachliche Realisierung der dazu gehörigen Subscriptiones in der Regel direkt semantisch eingeschränkt wird. Allerdings sind mit dieser höheren semantischen Präzision gleichzeitig auch Möglichkeiten der Konkretisierung verbunden, die es erlauben, konkrete Aussagen vorzunehmen, Positionen zu widersprechen, Verantwortliche zu benennen oder Forderungen zu formulieren.

Im Hinblick auf konkrete politische Anschlussüberlegungen lässt sich damit festhalten, dass materielle Bilder sowohl für die positive wie für die negative Prozessierung von Emotionen notwendig sind. Voraussetzung hierfür ist jedoch, wie am Beispiel des Hungerstreiks am Rindermarkt gesehen, dass politische Akteure auch die medialen Möglichkeiten haben, um materielle Bilder so zu verbreiten, dass sie diskursiv wirksam werden. Diese Feststellung leitet über zu den diesen Beitrag abschließenden handlungspraktischen Anschlussüberlegungen. 


\subsection{Politische Anschlussüberlegungen}

Nachdem im Bisherigen zwei deutlich konträre Darstellungen ein und desselben politischen Ereignisses durch zwei große überregionale deutsche Zeitungen vorrangig aus empirischer Sicht betrachtet worden sind, soll es nun um eine diskurspraktische Auswertung dieser Befunde hinsichtlich des Einsatzes von materiellen und sprachlichen Bildern gehen. Prinzipiell wäre eine solche Auswertung sowohl zugunsten einer hegemonialen Position gegen den Hungerstreik (im vorliegenden Fall repräsentiert durch die Frankfurter Allgemeine Zeitung) wie aber auch eine antihegemoniale Positionierung zugunsten der hungerstreikenden Flüchtlinge (hier repräsentiert durch die Süddeutsche Zeitung) denkbar. Mir geht es jedoch - wie einleitend angekündigt - um die semiotische Auswertung der Befunde im Hinblick auf politische Gegenbewegungen aus dem Bereich der Flüchtlingshilfe.

Im Hinblick auf diese Zielgruppe hat sich zunächst einmal bestätigt, dass politische Auseinandersetzungen nicht ohne Bilder - weder ohne sprachliche noch materielle - auskommen. Diese Feststellung ist vor allem im Hinblick auf antihegemoniale Gruppen und den dort noch immer verbreiteten Glauben an die unumstößliche Macht des rationalen Arguments von entscheidender Bedeutung. Subscriptiones alleine bleiben emotional unterentwickelt, wenn sie nicht durch Bilder veranschaulicht und belegt werden, das haben die Strategien beider untersuchter Zeitungen - wenn auch aus umgekehrter Perspektive - unterstrichen. Dies gilt umso mehr, wenn, wie im vorliegenden Fall, die politische Auseinandersetzung einer Bevölkerungsgruppe gilt, die aufgrund ihrer konkreten gesellschaftlichen Situation bereits mit einer Vielzahl negativer bildlicher Vorstellungen verbunden ist.

Hier haben die Ergebnisse der bildlichen Strategie der Süddeutschen Zeitung zum einen unterstrichen, dass es darum geht, den anonymen und abstrakten Bildern massenhafter Flucht anschauliche Bilder konkreter Einzelschicksale entgegenzusetzen. In diesem Zusammenhang hat es sich als wichtig erwiesen, dass die Betroffenen bildlich-interaktiv Kontakt aufnehmen können, sodass sie als politisch selbständig handelnde Akteure (im vorliegenden Fall im Rahmen einer konkreten politischen Aktion) sprach-bildlich sichtbar werden.

Trotz dieses Befunds hat sich der Einsatz von Bildern im Rahmen der empirischen Beobachtungen mehrfach auch als problematisch erwiesen: Sowohl das sprachliche Bild der "Schwangeren" unter den Planen, die Erwähnung von Bobby Sands und Holger Meins durch die Flüchtlinge, die Differenzen zwischen scheinbar neutralen Bildern von Flüchtlingen (Abbildungen 4 und 5 der $F A Z$ ) als auch die Darstellung Khorasanis als "Sprecher" der Flüchtlinge hat deutlich werden lassen, dass Bilder nicht nur vage bzw. ambivalent sein können (wie das sprachliche Bild der Schwangeren), sondern dass sie darüber hinaus häufig bereits auf problematische Weise gesellschaftlich diskursiv besetzt sind, wie die mit den Namen Holger Meins und Bobby Sands verbundenen Bilder. An dieser Stelle deutet sich allerdings eine Differenzierung an: Während Bilder und Symbole, die im politischen Diskurs als absolute Negativsymbole bildlich verankert sind, medial kaum uninterpretiert werden können und somit vermieden werden müssen, geht es im Hinblick auf die Nutzung ambivalenter Bilder wie der "Schwangeren" eher darum, entsprechende Subscriptiones in der medial-politischen Kontroverse zu verankern. 
In diesem Sinne schien die bildliche Berichterstattung der Süddeutschen zumindest in den ersten Tagen des Streiks darauf abzuzielen, hegemonialen Bildern und Symbolen wie dem "Münchener Rindermarkt" oder dem Symbol der "Burg" (vgl. Abbildung 2) antihegemoniale Bilder wie die "Zelte der Hungerstreikenden" oder bebilderte Einzelschicksale entgegenzusetzen. Diese Strategie wäre durchaus ausweitbar gewesen im Sinne einer negativen Bewertung von Bildern von "schimpfenden Münchener Bürgern" bzw. "Hundertschaften der Polizei".

Deutlich schwieriger ist die Frage zu beantworten, anhand welcher Bilder Sprecher/innen von Flüchtlingsgruppen wie der der Hungerstreikenden so aufgebaut werden können, dass sie nicht - wie Khorasani - direkt als Negativsymbol diskursiv diskreditiert werden. Hier lässt sich im Hinblick auf den Hungerstreik vermuten, dass auch die Weigerung der Flüchtlinge und Khorasanis selbst, überhaupt eine/n Gruppensprecher/in zu benennen, zum Problem wurde. Diese Strategie, die im Zusammenhang mit der Aktion am Rindermarkt darin deutlich wurde, dass Khorasani sich selbst immer nur als "Bote" der Flüchtlinge bezeichnet hat, kommt dort an ihre Grenzen, wo im Rahmen politischer Verhandlungen oder medialer Darstellungen diskursiv formuliert werden muss, was genau die Forderungen sind, die es zu verhandeln gilt. Dieses Problem, das im vorliegenden Fall zusätzlich durch die fehlenden Sprachkenntnisse der Flüchtlinge überlagert wurde, ist - so viel ist am Ende dieses Beitrags deutlich - bildlich sicherlich nicht durch einen "bärtigen iranischen Kommunisten" als Sprecher zu lösen.

Allerdings deuten sich im Anschluss an das Beispiel der Familie Wahafi weitere Möglichkeiten an, die im Fall des Hungerstreiks jedoch nicht genutzt wurden: So wäre sowohl eine Frau als Sprecherin als auch eine geschlechtsgemischte Sprecher/innen/gruppe eine mögliche und bildlich darstellbare Alternative gewesen. Ob diese möglicherweise durch eine/n Deutschen hätte unterstützt werden sollen, muss hier offen bleiben.

Insgesamt lässt sich abschließend festhalten, dass der Hungerstreik am Münchener Rindermarkt gezeigt hat, dass der Erfolg antihegemonialer Aktionen in jedem Fall darauf angewiesen ist, dass es gelingt, solche Aktionen medial bildlich und symbolische positiv zu präsentieren. In diesem Zusammenhang waren die "Zelte der Hungerstreikenden" auf dem Rindermarkt im "Herzen von München" dank der medialen Unterstützung der Süddeutschen Zeitung eine sinnvolle Strategie, die Anschaulichkeit von Bildern nutzend politisch aktiv zu werden.

\section{Literatur}

Fix, Ulla (2001): "An-schauliche Wörter? Wörter im Dienst der 'Bildhaftigkeit', 'Bildlichkeit', 'Sinnlichkeit', 'Lebendigkeit', 'Gegenständlichkeit' von Texten". In: Barz, Irmhild/Fix, U1la/Lerchner, Gotthard (Hrsg.): Das Wort in Text und Wörterbuch. Stuttgart, Hirtzel: 9-22. (= Abhandlungen der Sächsischen Akademie der Wissenschaft zu Leipzig 76/4).

Foucault, Michel (1977): Die Ordnung des Diskurses. Frankfurt a. M.: Ullstein.

Jewitt, Carey/Oyama, Rumiko (2001): "Visual Meaning: a Social Semiotic Approach". In:

Van Leeuwen, Theo/Jewitt, Carey (Hrsg.): Handbook of Visual Analysis. London etc., Sage: $134-156$.

Link, Jürgen (1975): Die Struktur des literarischen Symbols. München: Fink.

Link, Jürgen (1983): Elementare Literatur und generative Diskursanalyse. München: Fink. 
Link, Jürgen: (1984a): "Über ein Modell synchroner Systeme von Kollektivsymbolen sowie seine Rolle bei der Diskurs-Konstitution". In: Link, Jürgen/Wülfing, Wulf (Hrsg.): Bewegung und Stillstand in Metaphern und Mythen. Stuttgart, Klett-Cotta: 63-92.

Link, Jürgen (1984b): "kollektivsymbolik und mediendiskurse. Zur aktuellen rage, wie subjektive aufrüstung funktioniert". kultuRRevolution 1: 6-20.

Link, Jürgen (1985): Literaturwissenschaftliche Grundbegriffe. München: Fink.

Link, Jürgen (1997): Versuche über den Normalismus. Wie Normalität produziert wird. Opladen: Westdeutscher Verlag.

Link, Jürgen/Parr, Rolf (1990): "Semiotische Diskursanalyse". In: Bogdal, Klaus-Michael (Hrsg.): Neue Literaturtheorien. Eine Einführung. Opladen, Westdeutscher Verlag: 107130.

Meier, Stefan (2005): "Zeichenlesen und Netzdiskurs - Überlegungen zu einer semiotischen Diskursanalyse multimodaler Kommunikation". In: Fraas, Claudia/Klemm, Michael (Hrsg.): Mediendiskurse. Bestandsaufnahme und Perspektiven. Frankfurt a. M. etc., Lang: 123-141.

Schmitz, Ulrich (2005): "Blind für Bilder. Warum sogar Sprachwissenschaftler auch Bilder betrachten müssen". Osnabrücker Beiträge zur Sprachtheorie 69: 187-227.

Stöckl, Hartmut (1998): "(Un-)Chaining the floating image. Methodische Überlegungen zu einem Beschreibungs- und Analysemodell für die Bild/Textverknüpfung aus linguistischer und semiotischer Perspektive". Kodikas/Code Ars Semeiotica 21/1-2: 75-98.

Stöck1, Hartmut (2004): Die Sprache im Bild - Das Bild in der Sprache. Zur Verknüpfung von Sprache und Bild im massenmedialen Text. Berlin/New York: de Gruyter.

Stöckl, Hartmut (2011): "Sprache-Bild-Texte lesen. Bausteine zur Methodik einer Grundkompetenz". Diekmannshenke, Hajo/Klemm, Michael/Stöck1, Hartmut (Hrsg.): Bildlinguistik. Theorien - Methoden - Fallbeispiele. Berlin, Erich Schmidt Verlag: 45-70.

\section{Korpusverzeichnis}

Bender, Justus (2013): "Hungern, bis der Antrag durch ist". FAZ. www.faz.net/aktuell/politik/inland/demonstrierende-asylbewerber-hungern-bis-der-antragdurch-ist-12312580.html [31.07.2013].

Glas, Andreas/ Schmidt, Thomas (2014): "Flüchtlinge beenden Protestcamp". SZ. www.sueddeutsche.de/muenchen/muenchen-fluechtlinge-beenden-protestcamp-1.2239321 [30.11.2014].

Kastner, Bernd (2013a): "Verzweiflung im eiskalten Burgen-Land". SZ. www.sueddeutsche.de/bayern/asylpolitik-verzweiflung-im-eiskalten-burgen-land1.1710137 [02.07.2013].

Kastner, Bernd (2013b): "Im Regen stehen gelassen". SZ. www.sueddeutsche.de/muenchen/ fluechtlinge-im-hungerstreik-im-regen-stehen-gelassen-1.1704956 [25.05.2013].

Kastner, Bernd (2013c): "Menschen zweiter Klasse". SZ. www.sueddeutsche.de/muenchen/ fluechtlinge-im-hungerstreik-menschen-zweiter-klasse-1.1705637 [25.05.2013].

Kastner, Bernd (2013d): "Heimatlos an der Isar". SZ. www.fluechtlingsratbayern.de/pressebericht/items/heimatlos-an-der-isar.html [26.06.2013]. 
s. n. (2013a): "Die Verzweifelten und die Wütenden". SZ. http://www.sueddeutsche.de /muenchen/fluechtlinge-im-hungerstreik-im-regen-stehen-gelassen-1.1704956-2 [25.05.2013].

s. n. (2013b): "Haderthauer fordert Abbruch des Hungerstreiks". SZ. www.sueddeutsche.de/muenchen/fluechtlinge-demonstrieren-in-muenchen-haderthauerfordert-abbruch-des-hunger-streiks-1.1706405 [26.06.2013].

Schäffer, Albert (2013a): "Ein bitterböses Spiel mit Schicksalen". FAZ. www.faz.net/aktuell/politik/wahl-in-bayern/hungerstreik-von-asylbewerbern-einbitterboeses-spiel-mit-schicksalen-12264362.html [29.06.2013].

Schäfer, Albert (2013b): $\quad$ "Polizei räumt Asylbewerbercamp". FAZ. www.faz.net/aktuell/politik/inland/polizei-raeumt-asylbewerbercamp-eine-schwierige-undungewoehnliche-lage-12265802.html [01.07.2013].

Wagner, Richard (2013): "Vorerst gerettet". FAZ. http:/www.faz.net/aktuell/politik/inland/ polizei-raeumt-asylbewerbercamp-vorerst-gerettet-12266136.html [01.07.2013].

\section{Abbildungsnachweis:}

Abbildung 1: "Münchener Rindermarkt" http://de.wikipedia.org/wiki/Datei:

Rindermarkt_Muenchen-1.jpg [05.11.2015].

Abbildung 2: SZ 25.05.2013, 08:53 Uhr.

http://polpix.sueddeutsche.com/bild/1.1704973.1372143004/860x860/fluechtlingehungerstreik.jpg [05.11.2015].

Abbildung 3: http://polpix.sueddeutsche.com/bild/1.1704412.

1402659131/860x860/fluechtlinge-hungerstreik.jpg [25.05.2013].

Abbildung 4 und 5: http://faz.net/aktuell/politik/wahl-in-bayern/hungerstreik-vonasylbewerbern-ein-bitterboeses-spiel-mit-schicksalen-12264362.html [29.06.2013].

Abbildung 6: www.sueddeutsche.de/muenchen/fluechtlinge-im-hungerstreik-menschenzweiter-klasse-1.1705637 [25.06.2013].

Abbildung 7:

http://polpix.sueddeutsche.com/polopoly_fs/1.1706068.1372261537!/httpImage/image.jpg _gen/derivatives/860x860/image.jpg [26.06.2013].

Abbildung 8-11: www.faz.net/aktuell/hungerstreik-muenchner-fluechtlingsprotest-nachgrosseinsatz-beendet-13289775/nach-dem-einsatz-der-polizei-13289790.html [02.07.2013].

Abbildung 12: SZ, 01.07.2013.

Abbildung 13: FAZ, 31.07.2013. 